\title{
SPATIAL HETEROCLINIC BIFURCATION \\ OF TIME PERIODIC SOLUTIONS \\ TO THE GINZBURG-LANDAU EQUATION
}

\author{
BY \\ H. DANG-VU (UFR 920, Mathématiques pures et appliquées, Université Pierre et Marie Curie, \\ 75252-Paris, France) \\ AND \\ C. DELCARTE (Laboratoire d'Informatique pour la Mécanique et les Sciences de l'Ingénieur \\ (LIMSI)-Université de Paris-Sud, 91405-Orsay, France)
}

\begin{abstract}
In this paper we study the spatial structure of the time periodic solutions to the Ginzburg-Landau equation in various configurations (supercritical and subcritical). We show that spatially periodic bursting solutions or spatially heteroclinic solutions can occur depending upon the values of the coefficients. As a consequence of this study, we obtain an exact solution to the nonlinear system of Kapitula and Maier-Paape [16]. We then show that near a spatially heteroclinic solution there is an extremely intricate complex of spatially unstable solutions and KAM surfaces.
\end{abstract}

1. Introduction. In its most general form the (cubic) Ginzburg-Landau equation reads (see, e.g., [15], [26], [16]):

$$
\frac{\partial \Psi}{\partial t}=\left(R-R_{c}\right) \Psi+\left(b_{1}+i c_{1}\right) \frac{\partial^{2} \Psi}{\partial x^{2}}-\left(b_{3}+i c_{3}\right)|\Psi|^{2} \Psi
$$

where $\Psi=\Psi(x, t)$ is a complex function of space and time and $R, R_{c}, b_{1}, c_{1}, b_{3}, c_{3}$ are real constants with $b_{1}>0$.

$R=R_{c}$ is the value at which the trivial solution $\Psi(x, t)=0$ bifurcates to a periodic solution (the Stokes solution)

$$
\Psi(x, t)=\rho e^{i(k x+\omega t)}
$$

with

$$
\begin{gathered}
\rho^{2}=\frac{\epsilon-b_{1} k^{2}}{b_{3}}, \quad \epsilon=R-R_{c} \\
\omega=-\frac{\epsilon c_{3}}{b_{3}}-\left(c_{1}-\frac{b_{1} c_{3}}{b_{3}}\right) k^{2} .
\end{gathered}
$$

Received February 17, 1998.

2000 Mathematics Subject Classification. Primary 35Q55, 34C37; Secondary 37D45, 34 C23.

Key words and phrases. Ginzburg-Landau equation, heteroclinic solution, KAM surface. 
The bifurcation is supercritical if $R>R_{c}, b_{3}>0$ and subcritical if $R<R_{c}, b_{3}<0$ [11], [18], [26]. This property plays an important role in the problem we are considering.

Ginzburg-Landau equations have been studied from a variety of points of view. Hocking and Stewartson [14], Sirovich and Newton [24], [21], Holmes [15], and Takáč [25] have studied the temporal evolution of (1.1) in various cases of the parameters (see also [6], $[16],[10],[27])$. Front solutions have been discussed, for the case of real coefficients, by Eckmann and Gallay [12] and Bricmont and Kupiainen [3]. Transitions to chaos have been studied by Keefe [17] and others [20], [6], [23]. In this paper we study time periodic solutions of the Ginzburg-Landau equation of the form

$$
\Psi(x, t)=A(x) e^{i \omega t} .
$$

In [14], Hocking and Stewartson derived exact heteroclinic solutions to (1.1) of the form

$$
\Psi(x, t)=A_{0} p(x) e^{i(k \log |p(x)|+\omega t)}
$$

where

$$
p(x)=\frac{1}{\cosh m x}
$$

with real quantities $A_{0}, k, m$ satisfying some suitable conditions. This is the solitary wave solution found in ref. [22]. Van Saarloos and Hohenberg [26] called it a pulse solution. These solutions have been studied by a number of authors (see, e.g., [9], [4],[18], [26] and the references therein). In Sec. 2 we point out that exact bursting solutions to (1.1) of the form (1.4) also exist with

$$
p(x)=\frac{1}{\cos m x}
$$

but with different constants $A_{0}, k, m$. In Sec. 3 we show that our results extend earlier work on heteroclinic solutions to the Ginzburg-Landau equation [6], [16]. In particular, using our Theorem 2 we show that the solution to the nonlinear system (3.9), found by Kapitula and Maier-Paape [16], is (3.8). Section 4 is concerned with bifurcations involving heteroclinic orbits. The bifurcation to chaos is described, as seen through numerical experiments. We present some elementary consequences of the occurrence of a heteroclinic bifurcation, especially in terms of the cascades of bifurcations that accompany them. A preliminary account of these results has already been reported [5].

\section{Exact spatially heteroclinic and bursting solutions.}

2.1. The supercritical case. Assuming that the bifurcation is supercritical: $R>R_{c}$ and $b_{3}>0$, then after rescaling, Eq. (1.1) becomes ([19], [21], [7], [8])

$$
\frac{\partial \Psi}{\partial t}=\Psi+\left(1+i \gamma_{1}\right) \frac{\partial^{2} \Psi}{\partial x^{2}}-\left(1+i \gamma_{3}\right)|\Psi|^{2} \Psi
$$

with $\gamma_{1}, \gamma_{3} \in \mathbb{R}$. Substituting (1.3) into (2.1) yields the following equation:

$$
A^{\prime \prime}(x)+g_{1} A(x)-g_{2}|A(x)|^{2} A(x)=0,
$$

where ()$^{\prime}$ denotes $d() / d x$ and

$$
g_{1}=\frac{1-i \omega}{1+i \gamma_{1}}, \quad g_{2}=\frac{1+i \gamma_{3}}{1+i \gamma_{1}} .
$$


It is convenient to represent $g_{1}$ and $g_{2}$ in the polar form

$$
g_{1}=\rho_{1} e^{i \theta_{1}}, \quad g_{2}=\rho_{2} e^{i \theta_{2}} .
$$

Then we have the following

Theorem 2.1. Assume that

$$
\tan \theta_{2}=\frac{3 k}{2-k^{2}}
$$

where

$$
k=k_{ \pm}\left(\theta_{1}\right)=\frac{ \pm 1-\cos \theta_{1}}{\sin \theta_{1}} .
$$

Then Eq. (2.2) possesses an exact solution

$$
A(x)=A_{0} p(x) e^{i k \log |p(x)|}
$$

with

$$
p(x)= \begin{cases}\frac{1}{\cos \left(m_{+} x\right)} & \text { if } \quad k=k_{+} \\ \frac{1}{\cosh \left(m_{-} x\right)} & \text { if } \quad k=k_{-}\end{cases}
$$

where

$$
\begin{aligned}
m_{ \pm}^{2} & = \pm \frac{\rho_{1} \cos \theta_{1}}{1-k_{ \pm}^{2}} \\
A_{0}^{2} & =\frac{3 \rho_{1} \sin \theta_{1}}{2 \rho_{2} \sin \theta_{2}}
\end{aligned}
$$

Proof. We first note that $k_{ \pm}$are roots of the quadratic equation

$$
\sin \theta_{1} k^{2}+2 \cos \theta_{1} k-\sin \theta_{1}=0 .
$$

For later use we point out that

$$
\begin{aligned}
& k_{+} k_{-}=-1 \\
& k_{+}^{2}<1, \quad k_{-}^{2}>1 \quad \text { if } \cos \theta_{1}>0 \\
& k_{+}^{2}>1, \quad k_{-}^{2}<1 \quad \text { if } \cos \theta_{1}<0
\end{aligned}
$$

From (2.7) we have

$$
A^{\prime \prime}(x)=(1+i k)\left[\frac{p^{\prime \prime}}{p}+i k \frac{p^{2}}{p^{2}}\right] A(x), \quad|A(x)|^{2}=A_{0}^{2} p^{2} .
$$

Inserting (2.13) into (2.2) yields

$$
\frac{p^{\prime \prime}}{p}-k^{2} \frac{p^{\prime 2}}{p^{2}}+i k\left[\frac{p^{\prime \prime}}{p}+\frac{p^{2}}{p^{2}}\right]+g_{1}-g_{2} A_{0}^{2} p^{2}=0 .
$$

a) For $k=k_{+}$: from (2.8), (2.14) gives

$$
\begin{gathered}
m_{+}^{2}\left\{\left(k_{+}^{2}-1\right) \cos ^{2}\left(m_{+} x\right)+2-k_{+}^{2}+i k_{+}\left[3-2 \cos ^{2}\left(m_{+} x\right)\right]\right\} \\
+\rho_{1} \cos \theta_{1} \cos ^{2}\left(m_{+} x\right)-\rho_{2} A_{0}^{2} \cos \theta_{2}+i\left[\rho_{1} \sin \theta_{1} \cos ^{2}\left(m_{+} x\right)-\rho_{2} A_{0}^{2} \sin \theta_{2}\right]=0 .
\end{gathered}
$$


Equating the coefficients of $\cos ^{2}\left(m_{+} x\right)$ to zero we obtain the four equations

$$
\begin{aligned}
m_{+}^{2}\left(1-k_{+}^{2}\right) & =\rho_{1} \cos \theta_{1}, \\
m_{+}^{2}\left(2-k_{+}^{2}\right) & =\rho_{2} A_{0}^{2} \cos \theta_{2}, \\
2 k_{+} m_{+}^{2} & =\rho_{1} \sin \theta_{1}, \\
3 k_{+} m_{+}^{2} & =\rho_{2} A_{0}^{2} \sin \theta_{2} .
\end{aligned}
$$

Using (2.16) and (2.18),

$$
\tan \theta_{2}=\frac{3 k_{+}}{2-k_{+}^{2}} .
$$

On the other hand, from (2.15) and (2.17) we have

$$
\sin \theta_{1} k_{+}^{2}+2 \cos \theta_{1} k_{+}-\sin \theta_{1}=0 .
$$

From (2.15) we have

$$
m_{+}^{2}=\frac{\rho_{1} \cos \theta_{1}}{\left(1-k_{+}^{2}\right)} \text {. }
$$

From (2.17) and (2.18) we have

$$
A_{0}^{2}=\frac{3 \rho_{1} \sin \theta_{1}}{2 \rho_{2} \sin \theta_{2}}
$$

b) For $k=k_{-}$: from (2.8), (2.14) gives

$$
m_{-}^{2}\left\{\left(1-k_{-}^{2}\right) \cosh ^{2}\left(m_{-} x\right)-2+k_{-}^{2}+i k_{-}\left[2 \cosh ^{2}\left(m_{-} x\right)-3\right]\right\}
$$

$+\rho_{1} \cos \theta_{1} \cosh ^{2}\left(m_{-} x\right)-\rho_{2} A_{0}^{2} \cos \theta_{2}+i\left[\rho_{1} \sin \theta_{1} \cosh ^{2}\left(m_{-} x\right)-\rho_{2} A_{0}^{2} \sin \theta_{2}\right]=0$.

Equating the coefficients of $\cosh ^{2}\left(m_{-} x\right)$ to zero we obtain the four equations

$$
\begin{aligned}
m_{-}^{2}\left(1-k_{-}^{2}\right) & =-\rho_{1} \cos \theta_{1}, \\
m_{-}^{2}\left(2-k_{-}^{2}\right) & =-\rho_{2} A_{0}^{2} \cos \theta_{2}, \\
2 k_{-} m_{-}^{2} & =-\rho_{1} \sin \theta_{1}, \\
3 k_{-} m_{-}^{2} & =-\rho_{2} A_{0}^{2} \sin \theta_{2} .
\end{aligned}
$$

This yields, through the same manipulations as above,

$$
\begin{gathered}
\tan \theta_{2}=\frac{3 k_{-}}{2-k_{-}^{2}}, \\
\sin \theta_{1} k_{-}^{2}+2 \cos \theta_{1} k_{-}-\sin \theta_{1}=0, \\
m_{-}^{2}=-\frac{\rho_{1} \cos \theta_{1}}{1-k_{-}^{2}}, \\
A_{0}^{2}=\frac{3 \rho_{1} \sin \theta_{1}}{2 \rho_{2} \sin \theta_{2}} .
\end{gathered}
$$

To complete the proof we need to verify the conditions

$$
\begin{aligned}
m_{ \pm}^{2} & = \pm \frac{\rho_{1} \cos \theta_{1}}{1-k_{ \pm}^{2}}>0 \\
A_{0}^{2} & =\frac{3 \rho_{1} \sin \theta_{1}}{2 \rho_{2} \sin \theta_{2}}>0 .
\end{aligned}
$$


From (2.12) it is easy to see that (2.31) holds. Next observe, from (2.5), that

$$
\theta_{2}=\arctan \frac{3 k}{2-k^{2}}(\bmod \pi)
$$

It follows that $A_{0}^{2}$ can always be made positive by taking an appropriate choice of $\theta_{2}$.

2.2. The subcritical case. Assuming that the bifurcation is subcritical: $R<R_{c}$ and $b_{3}<0$. Then rescaling, Eq. (1.1) becomes [24], [15], [16]

$$
\frac{\partial \Psi}{\partial t}=-\Psi+\left(1+i \beta_{1}\right) \frac{\partial^{2} \Psi}{\partial x^{2}}+\left(1+i \beta_{3}\right)|\Psi|^{2} \Psi,
$$

with $\beta_{1}, \beta_{3} \in \mathbb{R}$. Substituting (1.3) into (2.34) we get the following equation:

$$
A^{\prime \prime}(x)-g_{1} A(x)+g_{2}|A(x)|^{2} A(x)=0,
$$

with

$$
g_{1}=\frac{1+i \omega}{1+i \beta_{1}}=\rho_{1} e^{i \theta_{1}}, \quad g_{2}=\frac{1+i \beta_{3}}{1+i \beta_{1}}=\rho_{2} e^{i \theta_{2}} .
$$

Then we have the following

Theorem 2.2. Assume that

$$
\tan \theta_{2}=\frac{3 k}{2-k^{2}}
$$

where

$$
k=k_{ \pm}\left(\theta_{1}\right)=\frac{ \pm 1-\cos \theta_{1}}{\sin \theta_{1}} .
$$

Then Eq. (2.35) possesses an exact solution

$$
A(x)=A_{0} p(x) e^{i k \log |p(x)|}
$$

with

$$
p(x)= \begin{cases}\frac{1}{\cosh \left(m_{+} x\right)} & \text { if } \quad k=k_{+} \\ \frac{1}{\cos \left(m_{-} x\right)} & \text { if } \quad k=k_{-}\end{cases}
$$

where

$$
\begin{aligned}
m_{ \pm}^{2} & = \pm \frac{\rho_{1} \cos \theta_{1}}{1-k_{ \pm}^{2}} \\
A_{0}^{2} & =\frac{3 \rho_{1} \sin \theta_{1}}{2 \rho_{2} \sin \theta_{2}}
\end{aligned}
$$

The proof is carried out in the same way as for Theorem 1.

REMARK 2.3. Substituting (2.38) (or (2.6)) into (2.37) (or (2.5)) we then obtain

$$
\tan \theta_{2}=\frac{ \pm 3 \sin \theta_{1}}{1 \pm 3 \cos \theta_{1}}
$$


3. Previous work. In [6] a study of the solutions of (2.1) was begun by taking

$$
\gamma_{1}=\epsilon a, \quad \gamma_{3}=\epsilon b, \quad \Psi(x, t)=\rho(x) e^{i[\theta(x)-\epsilon w t]}
$$

with $a, b \in \mathbb{R}, 0<\epsilon \ll 1$. If we write

$$
V=\rho^{\prime}, \quad \Omega=\rho^{2} \theta^{\prime}, \quad()^{\prime}=d() / d x,
$$

then by substituting into (2.1) we get the following set of equations:

$$
\begin{aligned}
\rho^{\prime} & =V \\
V^{\prime} & =-\rho+\rho^{3}+\frac{\Omega^{2}}{\rho^{3}}+\frac{a \epsilon^{2}}{1+\epsilon^{2} a^{2}} \rho\left[(a-w)-\rho^{2}(a-b)\right] \\
\Omega^{\prime} & =\frac{\epsilon}{1+\epsilon^{2} a^{2}} \rho^{2}\left[(a-w)-\rho^{2}(a-b)\right] .
\end{aligned}
$$

Upon comparison with (2.2) we get

$$
\begin{aligned}
\omega & =-\epsilon w \\
g_{1} & =\frac{1+i \epsilon w}{1+i \epsilon a}=\rho_{1} e^{\theta_{1}} \\
g_{2} & =\frac{1+i \epsilon b}{1+i \epsilon a}=\rho_{2} e^{\theta_{2}} .
\end{aligned}
$$

It follows that

$$
\begin{array}{ll}
\rho_{1}=\sqrt{\frac{1+\epsilon^{2} w^{2}}{1+\epsilon^{2} a^{2}}}, & \tan \theta_{1}=\frac{\epsilon(w-a)}{1+\epsilon^{2} w a}, \\
\rho_{2}=\sqrt{\frac{1+\epsilon^{2} b^{2}}{1+\epsilon^{2} a^{2}}}, \quad \tan \theta_{1}=\frac{\epsilon(b-a)}{1+\epsilon^{2} b a} .
\end{array}
$$

Thus we find the following solution for (3.1):

$$
\rho(x)=A_{0} p(x), \quad V=A_{0} p^{\prime}(x), \quad \Omega=A_{0}^{2} k p(x) p^{\prime}(x),
$$

with $k, p(x), A_{0}$ given by $(2.6),(2.8),(2.10)$ respectively. It is easy to see that for $k=k_{-}$, the solution (3.4) is a homoclinic orbit in the phase space $(\rho, V, \Omega)$ connecting the origin to itself:

$$
\lim _{x \rightarrow \pm \infty}(\rho, V, \Omega)=(0,0,0) .
$$

In ref. [16] Kapitula and Maier-Paape studied Eq. (2.35) by taking

$$
\beta_{1}=0, \quad \omega=\sigma, \quad \beta_{3}=q
$$

with $\sigma, q \in \mathbb{R}$. Upon comparison with (2.36) we find

$$
\begin{aligned}
& g_{1}=1+i \sigma=\rho_{1} e^{\theta_{1}}, \\
& g_{2}=1+i q=\rho_{2} e^{\theta_{2}} .
\end{aligned}
$$

It follows that

$$
\sigma=\tan \theta_{1}, \quad q=\tan \theta_{2} .
$$

Substituting $k=k_{+}=\left(1-\cos \theta_{1}\right) / \sin \theta_{1}$ into (2.37) we obtain

$$
\tan \theta_{2}=\frac{3 \sin \theta_{1}}{1+3 \cos \theta_{1}}
$$


(cf. Remark 2.3). Equations (3.5) and (3.6) are combined to give

$$
q=\frac{\sigma}{1+\frac{1}{3} \sqrt{1+\sigma^{2}}} .
$$

This is the pulse condition found in ref. [16] using Hocking-Stewartson's method [14]. Notice that our system (2.23)-(2.26) is similar to the system (5.3) of ref. [14]. Notice also that, using the notation of ref. [16],

$$
\begin{aligned}
A(x) & =\rho(x) e^{\int^{x} \phi(s) d s}, \\
u(x) & =\frac{\rho^{\prime}(x)}{\rho(x)},
\end{aligned}
$$

it is easy to show that, in the phase space $(\rho, u, \phi)$, the pulse solution

$$
\begin{aligned}
A(x) & =A_{0} p(x) e^{i k_{+} \log |p(x)|}, \\
p(x) & =\frac{1}{\cosh \left(m_{+} x\right)}
\end{aligned}
$$

of Theorem 2 is a heteroclinic orbit connecting the two equilibria $\left(0, u_{+}, \phi_{+}\right)$and $\left(0, u_{-}, \phi_{-}\right)$with

$$
\begin{aligned}
& u_{ \pm}=\lim _{x \rightarrow \mp \infty} u(x)= \pm m_{+}=\frac{ \pm \sigma}{\sqrt{2\left(\sqrt{1+\sigma^{2}}-1\right)}}, \\
& \phi_{ \pm}=\lim _{x \rightarrow \mp \infty} \phi(x)= \pm k_{+} m_{+}= \pm \sqrt{\frac{\sqrt{1+\sigma^{2}}-1}{2}} .
\end{aligned}
$$

It is easy to check that $u_{+}$is the positive solution and $u_{-}$is the negative solution to the system

$$
\begin{aligned}
& u_{ \pm}^{2}-\phi_{ \pm}^{2}-1=0 \\
& \sigma-2 u_{ \pm} \phi_{ \pm}=0
\end{aligned}
$$

of Lemma 2.4 of ref. [16].

4. Reduction to the third order. We now return to Eq. (2.35) and introduce a transformation first suggested by Sirovich and Newton [24] and Holmes [15]:

$$
A(x)=\rho(x) e^{i W(x)}, \quad r=\rho^{2}, \quad v=\frac{\rho^{\prime}}{\rho}, \quad w=W^{\prime} .
$$

Direct substitution then shows that Eq. (2.35) becomes a third-order system:

$$
\begin{aligned}
r^{\prime} & =2 r v, \\
v^{\prime} & =w^{2}-v^{2}+\rho_{1} \cos \theta_{1}-r \rho_{2} \cos \theta_{2}, \\
w^{\prime} & =-2 v w+\rho_{1} \sin \theta_{1}-r \rho_{2} \sin \theta_{2} .
\end{aligned}
$$

The linearized system corresponding to (4.2) has a Jacobian matrix

$$
J=\left[\begin{array}{ccc}
2 v & 2 r & 0 \\
-\rho_{2} \cos \theta_{2} & -2 v & 2 w \\
-\rho_{2} \sin \theta_{2} & -2 w & -2 v
\end{array}\right] .
$$

The flow defined by $(4.2)$ is volume preserving in $\left(r^{2}, v, w\right)$ space, i.e.,

$$
\frac{\partial\left(r^{2}\right)^{\prime}}{\partial r^{2}}+\frac{\partial(v)^{\prime}}{\partial v}+\frac{\partial(w)^{\prime}}{\partial w}=0
$$


The fixed points in (4.2) are found by setting the right-hand sides of (4.2) to zero. In the phase space $(r, v, w)$ the heteroclinic orbit (3.7) connects the two fixed points $\left(0, v_{ \pm}, w_{ \pm}\right)$, corresponding to $\left(0, u_{ \pm}, \phi_{ \pm}\right)$in ref. [16] or $L_{ \pm}$in ref. [26], defined by

$$
v_{ \pm}= \pm m_{+}, \quad w_{ \pm}= \pm k_{+} m_{+} .
$$

$\left(v_{ \pm}, w_{ \pm}\right)$are solutions of the system

$$
\begin{aligned}
& w^{2}-v^{2}+\rho_{1} \cos \theta_{1}=0, \\
& -2 v w+\rho_{1} \sin \theta_{1}=0 .
\end{aligned}
$$

If $\theta_{1}>\theta_{2}$ and $0<\theta_{1}, \theta_{2}<\pi / 2$, then the system (4.2) has two other fixed points, corresponding to $N_{ \pm}$in ref. [26],

$$
r_{0}=\frac{\rho_{1} \sin \theta_{1}}{\rho_{2} \sin \theta_{2}}, \quad v_{0}=0, \quad w_{0}= \pm \sqrt{\frac{\rho_{1} \sin \left(\theta_{1}-\theta_{2}\right)}{\sin \theta_{2}}} .
$$

These fixed points correspond to the plane wave solutions of $(2.35)$ :

$$
A(x)=\rho e^{i \int^{x} w(s) d s}=\sqrt{r_{0}} e^{i\left(w_{0} x+c\right)}, \quad c=\text { const. }
$$

Thus the number of fixed points is $\leq 4$, as expected [18], [26]. Their stability is obtained by studying the sign of the real part of the eigenvalues of $J$ (see, e.g., [26] and [16]). The rest of this section is devoted to the study of the dynamics near the heteroclinic solution. Figures 1(a) - 6 illustrate the results with an example:

$$
\begin{aligned}
& \theta_{1}=\theta_{1}^{*}=\pi / 8 \\
& \theta_{2}=\theta_{2}^{*}=\arctan \frac{3 k_{+}\left(\theta_{1}^{*}\right)}{2-k_{+}^{2}\left(\theta_{1}^{*}\right)}=\arctan \frac{3 \sin \theta_{1}^{*}}{1+3 \cos \theta_{1}^{*}}=0.295479 \ldots, \\
& \rho_{1}=\rho_{2}=1,
\end{aligned}
$$

corresponding approximately to the study by Sirovich and Newton [24]. For $\theta_{1}=\theta_{1}^{*}$, $\theta_{2}=\theta_{2}^{*}, r=r^{*}=A_{0}^{2}$, the heteroclinic orbit is indeed found. In Fig. 1(a) we trace the heteroclinic orbit in the $\left(\rho, \rho^{\prime}\right)$ plane with $\rho(x)=|A(x)|$. We observe that there exists a hyperbolic saddle at $(0,0)$ with a homoclinic orbit $C$; points on $C$ approach $(0,0)$ for both $x \rightarrow+\infty$ and $x \rightarrow-\infty$;

$$
\lim _{x \rightarrow \pm \infty} \rho(x)=\lim _{x \rightarrow \pm \infty} \rho^{\prime}(x)=0 .
$$

Figure 1(b) shows the projection of the orbit onto the $(r, v)$ plane. Note that in the phase space $(r, v, w)$, points on the orbit approach $\left(0, u_{+}, w_{+}\right)$for $x \rightarrow-\infty$ and $\left(0, u_{-}, w_{-}\right)$for $x \rightarrow+\infty$.

If we start orbits close to the heteroclinic orbit, we find an infinite number of unstable orbits. We will use the Poincaré section technique to reduce the recorded trajectory points to a two-dimensional plane. Figures $3-6$ show the Poincaré sections at $v=\rho^{\prime}=0, v^{\prime}<0$. A number of integrations of $(4.2)$, with $\left(\theta_{1}, \theta_{2}\right) \approx\left(\theta_{1}^{*}, \theta_{2}^{*}\right)$, were performed, for various initial values used to generate a variety of trajectories. The solutions were completely different from those obtained with $\left(\theta_{1}, \theta_{2}\right)=\left(\theta_{1}^{*}, \theta_{2}^{*}\right)$. The sections $v=0\left(v^{\prime}<0\right)$ are covered with the intersections of (somewhat deformed) tori which signal quasiperiodic motions (or KAM surfaces). Notice that the six elliptical curves of Fig. 3, for example, are generated by a single trajectory. These curves can be thought of as the intersections with the Poincaré plane $v=0, v^{\prime}<0$ of a torus that winds around the main inner KAM 


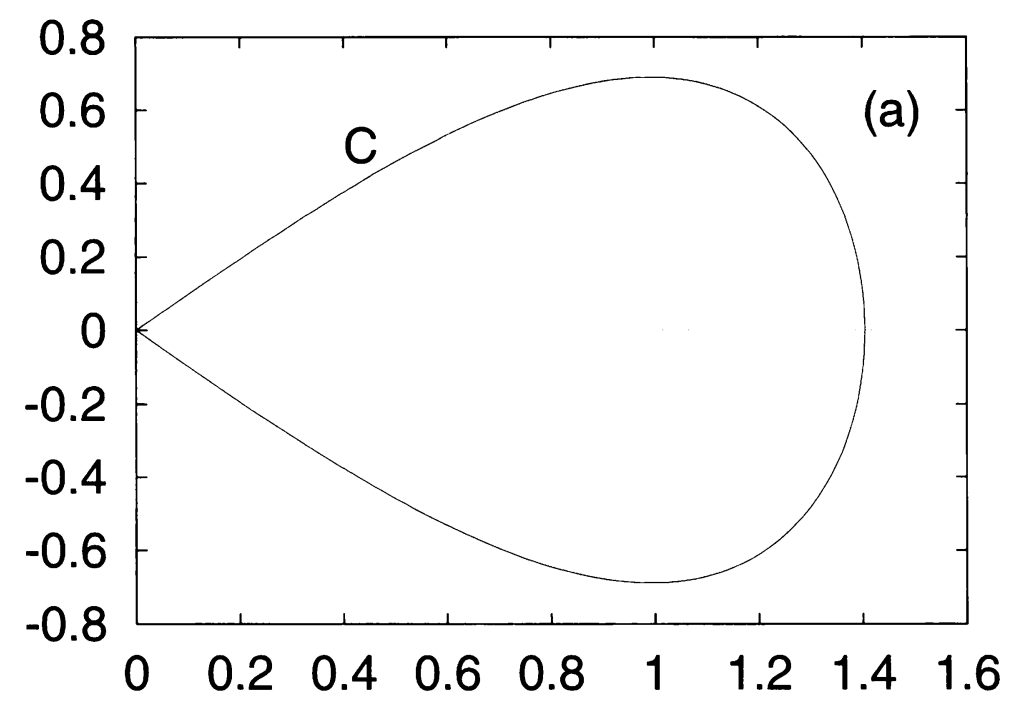

FIG. 1(a). Heteroclinic solution at $\theta_{1}=\theta_{1}^{*}=\pi / 8, \theta_{2}=\theta_{2}^{*}=$ $\arctan \left[3 \sin \theta_{1}^{*} /\left(1+3 \cos \theta_{1}^{*}\right)\right]=0.295479 \ldots, \rho_{1}=\rho_{2}=1 ;$ projection of the solution onto the $\left(\rho, \rho^{\prime}\right)$ plane

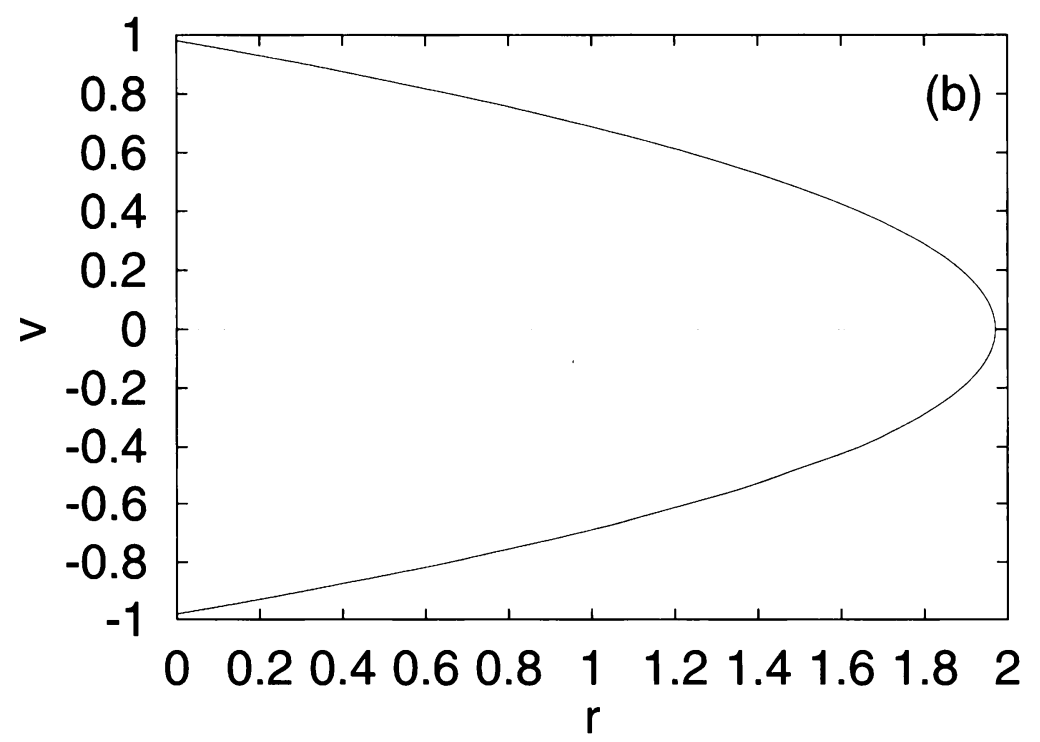

FIG. 1(b). Heteroclinic solution at $\theta_{1}=\theta_{1}^{*}=\pi / 8, \theta_{2}=\theta_{2}^{*}=$ $\arctan \left[3 \sin \theta_{1}^{*} /\left(1+3 \cos \theta_{1}^{*}\right)\right]=0.295479 \ldots, \rho_{1}=\rho_{2}=1 ;$ projection of the solution onto the $(r, v)$ plane

torus six times before closing. As we move outwards in Fig. 3 we obtain an island chain (labeled by IC) of eight elliptic points and eight hyperbolic points. The smeared parts of the Poincaré section near the hyperbolic points indicate that this orbit is chaotic. 


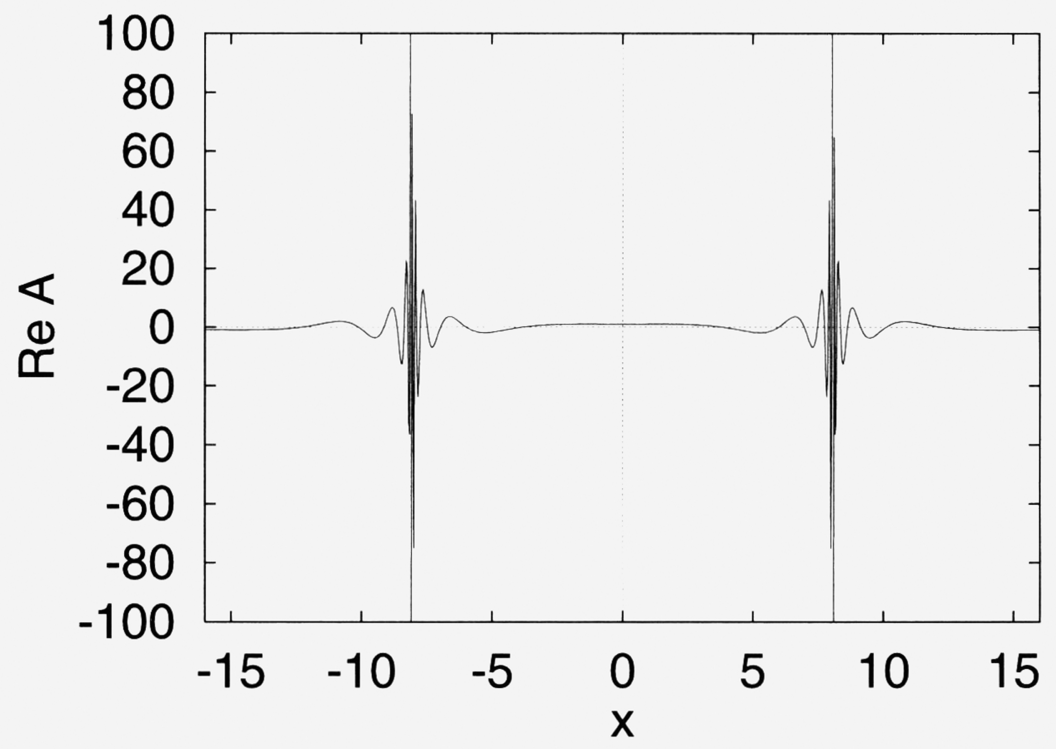

FIG. 2(a). Bursting solution at $\theta_{1}=\theta_{1}^{*}=\pi / 8, \theta_{2}=$ $-\arctan \left[3 \sin \theta_{1}^{*} /\left(1-3 \cos \theta_{1}^{*}\right)\right]=0.574979 \ldots, \rho_{1}=\rho_{2}=1$ obtained by plotting $\operatorname{Re} A(x)$ against $x\left(-\pi / m_{-} \leq x \leq \pi / m_{-}\right)$

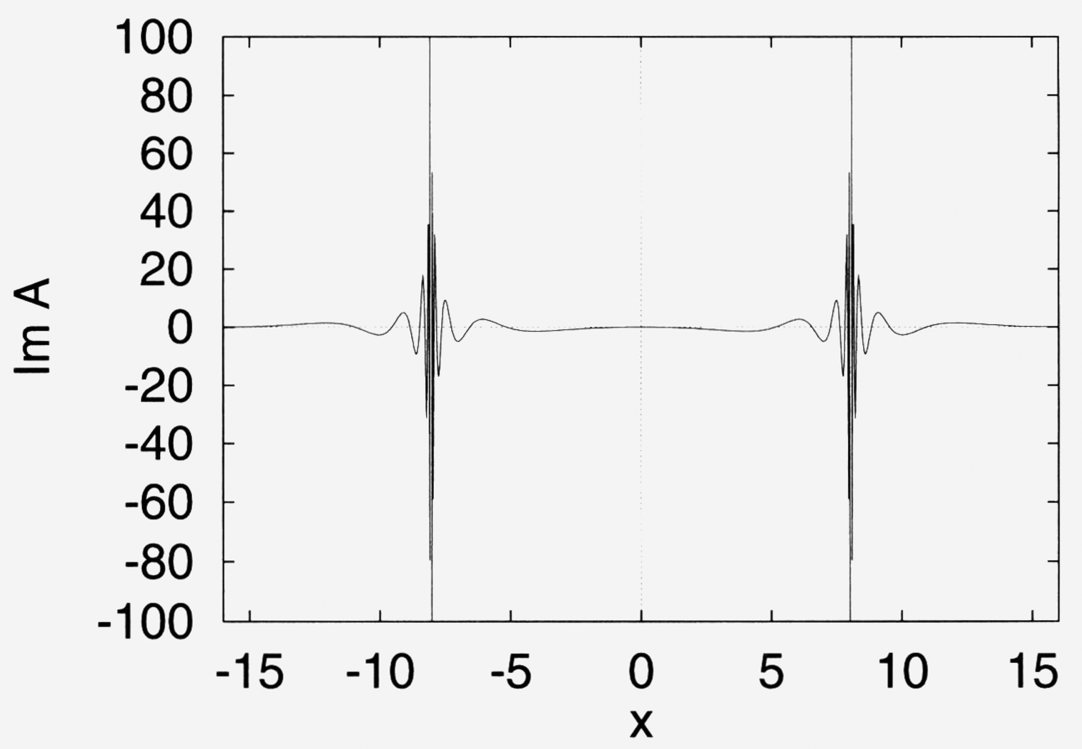

FIG. 2(b). Bursting solution at $\theta_{1}=\theta_{1}^{*}=\pi / 8, \theta_{2}=$ $-\arctan \left[3 \sin \theta_{1}^{*} /\left(1-3 \cos \theta_{1}^{*}\right)\right]=0.574979 \ldots, \rho_{1}=\rho_{2}=1$ obtained by plotting $\operatorname{Im} A(x)$ against $x\left(-\pi / m_{-} \leq x \leq \pi / m_{-}\right)$ 


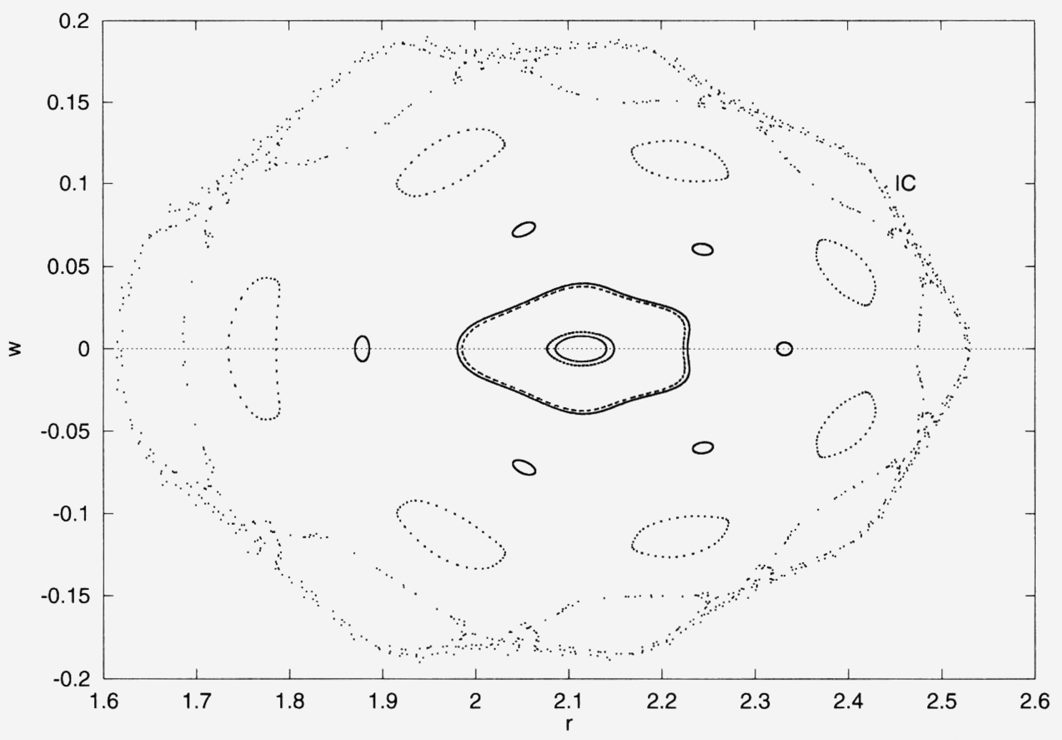

FIG. 3. Poincaré section $v=0, v^{\prime}<0$ at $\theta_{1}=\theta_{1}^{*}, \theta_{2}=\theta_{2}^{*}-0.03$, $\rho_{1}=\rho_{2}=1$. Orbits started with $v=0, w=0, r=r^{*}, r=r^{*} \pm 0.1$, $r=r^{*} \pm 0.2$ where $r^{*}=A_{0}^{2}$.

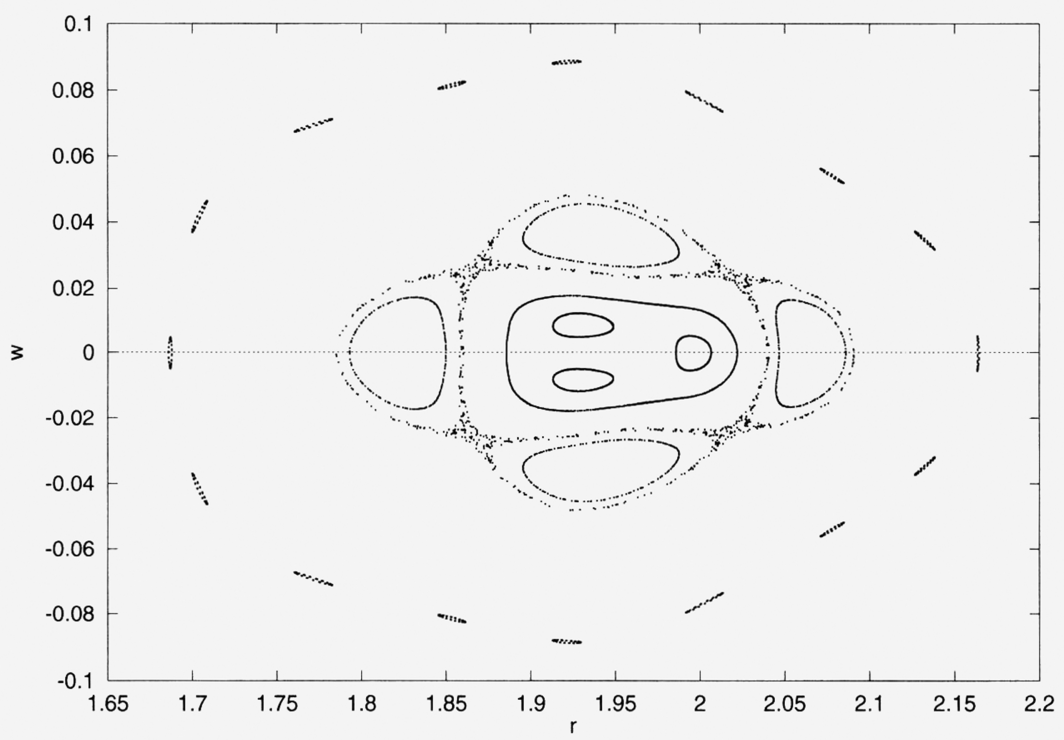

FIG. 4. Poincaré section $v=0, v^{\prime}<0$ at $\theta_{1}=\theta_{1}^{*}+0.02, \theta_{2}=$ $\theta_{2}^{*}+0.02, \rho_{1}=\rho_{2}=1$. Orbits started with $v=0, w=0, r=r^{*}$, $r=r^{*} \pm 0.1, r=r^{*} \pm 0.2$ where $r^{*}=A_{0}^{2}$. 


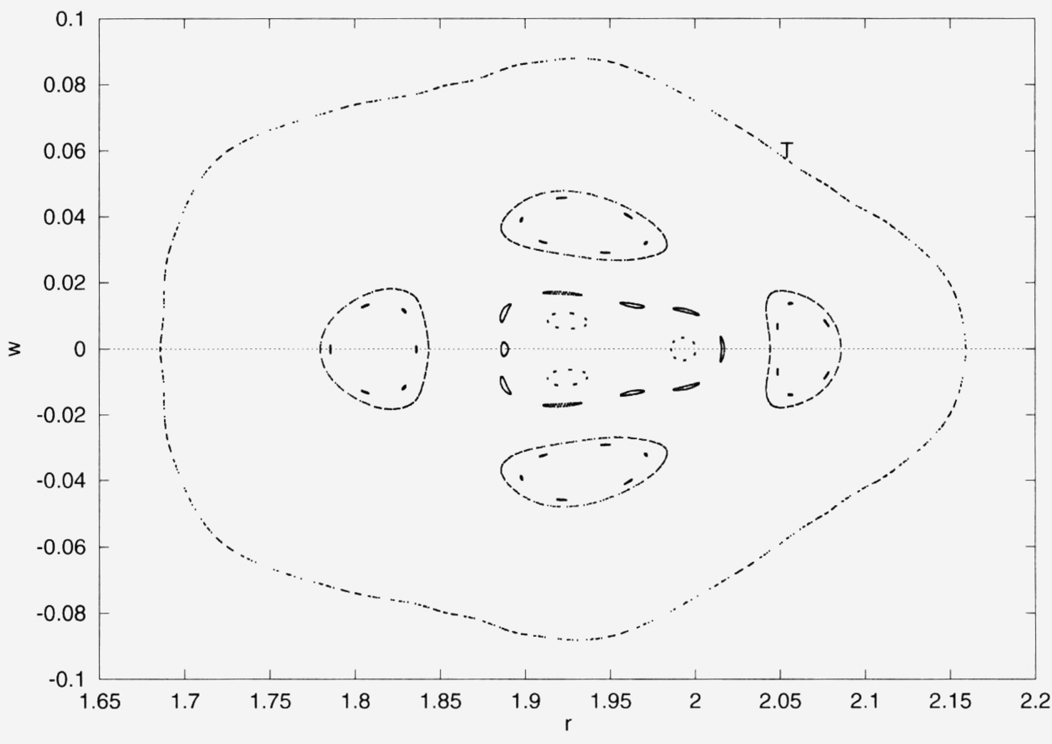

FIG. 5. Poincaré section $v=0, v^{\prime}<0$ at $\theta_{1}=\theta_{1}^{*}+0.025, \theta_{2}=$ $\theta_{2}^{*}+0.025$. $\rho_{1}=\rho_{2}=1$. Orbits started with $v=0, w=0, r=r^{*}$, $r=r^{*} \pm 0.1, r=r^{*} \pm 0.2$ where $r^{*}=A_{0}^{2}$.

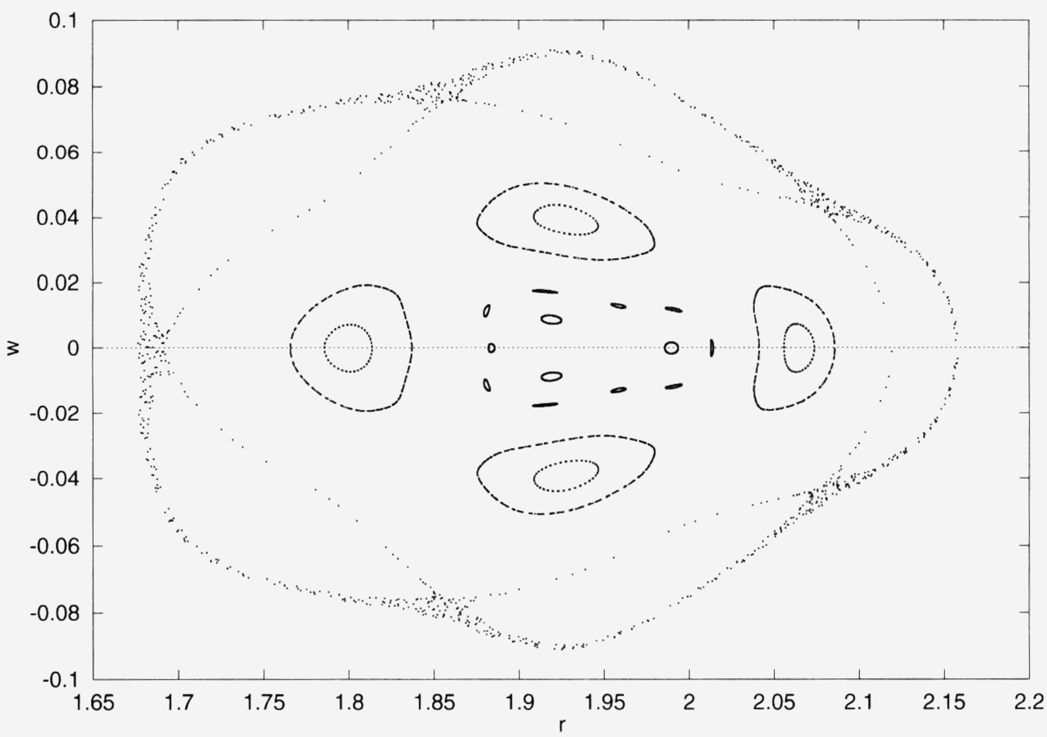

FIG. 6. Poincaré section $v=0, v^{\prime}<0$ at $\theta_{1}=\theta_{1}^{*}+0.03, \theta_{2}=$ $\theta_{2}^{*}+0.03, \rho_{1}=\rho_{2}=1$. Orbits started with $v=0, w=0, r=r^{*}$, $r=r^{*} \pm 0.1, r=r^{*} \pm 0.2$ where $r^{*}=A_{0}^{2}$. 
Features resembling island chains are also apparent in Fig. 4 and Fig. 6. The interesting point is that in Fig. 5 an invariant torus (labeled by $\mathbf{T}$ ) has dissolved and we obtain in Fig. 6 an island chain of five elliptic points and five hyperbolic points. Note the complexity of the island structures and the clearly chaotic orbits.

5. Conclusion. In this brief note we have given a relatively simple proof that the Ginzburg-Landau equation possesses a class of spatially heteroclinic solutions and a class of spatially periodic bursting solutions. Using the Poincaré section technique, we detected the occurrence of island chains following the heteroclinic bifurcation. These island chains are just the structure expected from the Poincaré-Birkhoff theorem [2] and have been seen in many mathematical models [13], [1].

Acknowledgments. Computational resources for this work were provided by the Université de Paris-Sud, Orsay. A preliminary account of this work was presented at the International Conference on Nonlinear Dynamics, Chaotic and Complex Systems held in Zakopane, Poland, 7-12 November 1995, under the auspices of the Directorate General XII, EC and the Polish Academy of Sciences. The first author would like to express his sincere thanks to professor R. Zelazny for financial support and hospitality at the Conference.

\section{REFERENCES}

[1] V. I. Arnold, Mathematical Methods in Classical Mechanics, Springer-Verlag, New York, 1978

[2] G. D. Birkhoff, Nouvelles recherches sur les systèmes dynamiques, Mem. Pont. Acad. Sci. Novi Lyncei 1, 85-216 (1935)

[3] J. Bricmont and A. Kupiainen, Stability of moving fronts in the Ginzburg-Landau equation, Comm. Math. Phys. 159, 287-318 (1994)

[4] F. Cariello and M. Tabor, Painlevé expansions for nonintegrable evolution equations, Physica D 39, 77-94 (1989)

[5] H. Dang-Vu and C. Delcarte, Bifurcations and chaos in the steady-state solutions of the GinzburgLandau equation in: "International Conference on Nonlinear Dynamics, Chaotic and Complex Systems", Zakopane, Poland, 7-12 November, 1995, Polish Scientific Publishers PWN, Warszawa, 1996, pp. 317-325

[6] A. Doelman, Slow time-periodic solutions of the Ginzburg-Landau equation, Physica D 40, 156-172 (1989)

[7] A. Doelman, Finite-dimensional models of the Ginzburg-Landau equation, Nonlinearity 4, 231-250 (1991)

[8] A. Doelman, Traveling waves in the complex Ginzburg-Landau equation, J. Nonlinear Sci., 3, 225266 (1993)

[9] A. Doelman, Breaking the hidden symmetry in the Ginzburg-Landau equation, Physica D 97, 398428 (1996)

[10] C. R. Doering, J. D. Gibbon, D. D. Holm, and B. Nicolaenko, Low dimensional behaviour in the complex Ginzburg-Landau equations, Nonlinearity 1, 279-309 (1988)

[11] W. Eckhaus, The Ginzburg-Landau manifold is an attractor, J. Nonlinear Sci. 3, 329-348 (1993)

[12] J.-P. Eckmann and Th. Gallay, Front solutions for the Ginzburg-Landau equation, Comm. Math. Phys. 152, 221-248 (1993)

[13] M. Hénon, The applicability of the third integral of motion: Some numerical experiments, Astrophys. J. 69, 73-79 (1964)

[14] L. M. Hocking and K. Stewartson, On the nonlinear response of a marginally unstable plane parallel flow to a two-dimensional disturbance, Proc. Roy. Soc. London 326A, 289-313 (1972)

[15] Ph. Holmes, Spatial structure of time-periodic solutions of the Ginzburg-Landau equation, Physica D 23, 84-90 (1986) 
[16] T. Kapitula and S. Maier-Paape, Spatial dynamics of time periodic solutions for the GinzburgLandau equation, Z. angew. Math. Phys. (ZAMP) 47, 265-305 (1996)

[17] L. R. Keefe, Dynamics of perturbed wavetrain solutions to Ginzburg-Landau equation, Stud. Appl. Math. 73, 91-153 (1985)

[18] M. Landman, Solutions of the Ginzburg-Landau equation of interest in shear flow transition, Stud. Appl. Math. 76, 187-237 (1987)

[19] B. J. Matkowsky and V. Volpert, Stability of plane wave solutions of complex Ginzburg-Landau equations, Quart. Appl. Math. 51, 265-281 (1993)

[20] H. T. Moon, P. Huerre, and L. G. Redekopp, Transitions to chaos in the Ginzburg-Landau equation, Physica D 7, 135-152 (1983)

[21] P. K. Newton and L. Sirovich, Instabilities of the Ginzburg-Landau equation: Periodic solutions, Quart. Appl. Math. 44, 49-58 (1986)

[22] K. Nozaki and N. Bekki, Exact solutions of the generalized Ginzburg-Landau equation, J. Phys. Soc. Jap. 53, 1581-1582 (1984)

[23] B. I. Shraiman, A. Pumir, W. van Saarloos, P. C. Hohenberg, H. Chaté, and M. Holen, Spatiotemporal chaos in the one-dimensional complex Ginzburg-Landau equation, Physica D 57, 241-248 (1992)

[24] L. Sirovich and P. K. Newton, Periodic solutions of the Ginzburg-Landau equation, Physica D 21, 115-125 (1986)

[25] P. Takáč, Invariant 2-tori in the time-dependent Ginzburg-Landau equation, Nonlinearity 5, 289-321 (1992)

[26] W. van Saarloos and P. C. Hohenberg, Fronts, pulses, sources and sinks in generalized complex Ginzburg-Landau equations, Physica D 56, 303-367 (1992)

[27] Y. Yang, Global spatially periodic solutions to the Ginzburg-Landau equation, Proc. Roy. Soc. Edinburgh A 110, 263-273 (1988) 